\title{
Sensory and Organoleptic Cup Attributes of Robusta Coffee (Coffea canephora Pierre ex A. Froehner)
}

\author{
Aluka P. (Corresponding Author)
}

National Agricultural Research Organization (NARO), National Coffee Resources Research Institute (NaCORRI), P.O. Box 185 Mukono, Uganda. Email: p.aluka@yahoo.com

Davrieu F., \& Fourny G.

UMR AGAP, CIRAD, 34398 Montpellier, France

Ngugi K.

Department of Plant Sciences and Crop Protection, Faculty of Agriculture, College of Agriculture and Veterinary Sciences, University of Nairobi, P.O Box 30197-00100, Nairobi, Kenya

Bakomeza F., Neumbe B., Kyamuhangire R., \& Ngabirano H.

Uganda Coffee Development Authority (UCDA), P.O. Box 7267 Kampala, Uganda

Received: August 31, 2015 Accepted: September 16, 2015 Published: December 28, 2015

doi:10.5296/jas.v4i1.8789

URL: http://dx.doi.org/10.5296/jas.v4i1.8789

\begin{abstract}
Coffea canephora organoleptic cup attributes are the most important factors that define its price in world markets. Determining the components that contribute to the diversity of organoleptic characters will help in the improvement of these qualities in order to obtain favourable markets. Two hundred and six genotypes from twenty one districts and two research institutes were analyzed by a three member expert panel from Uganda Coffee Development Authority using a 10 point descriptive scale and protocols from, The Coffee Quality Institute of America (CQIA). The results revealed that the evaluators' organoleptic cup trait ratings were significantly different $(\mathrm{p}<0.05)$ for all attributes, reflecting a diversity of cup interests. Four multivariate groups that were significantly different for fragrance, aroma and flavour were formed offering diverse cup tests to different markets. A variety of
\end{abstract}


fine and commercial flavours were detected in ripe cherry and green roasted beans. Cup balance contributed the highest regression coefficient $\left(\mathrm{R}^{2}=0.90\right)$ to overall assessment while fragrance/aroma had the least $\left(\mathrm{R}^{2}=0.22\right)$. The above average rating of $75 \%$ for cup balance, flavour, mouth feel, aftertaste, fragrance and aroma revealed that Ugandan Robusta coffees were of high quality with a mild taste. The higher cup acidity among land races, 'nganda' and 'erecta' genotypes revealed that genotypes with high sugars and cup acidity could be selected for from local germ-plasm. Coffee types and environmental factors such as soil texture, altitude and location influenced the content and level of organoleptic cup attributes. A diversity of flavours that exist among Uganda Robusta coffee and has so far remained unexploited, would provide new marketing channels, enhance quality and earn the country the much desired foreign exchange capital.

Keywords, Acidity, Aroma, Bitterness, Cup balance, Cup profiles

\section{Introduction}

Coffee being the most traded agricultural crop in the world has several quality classification systems aimed at facilitating the market and value addition (Leroy et al., 2006a). Coffee quality may be defined by widely varying characteristics such as physical appearance, moisture content and organoleptic measurements. Organoleptic qualities are the most difficult to define because they are based on consumer perception of subjective and sensory factors of fragrance, aroma, taste and flavours. Two types of analyses have been used to describe sensory evaluation; the hedonic organoleptic cup evaluation which is done by 60 assessors representing a population sample where the preference is sought (Leroy et al., 2006a) and the ' descriptive analysis' where trained assessors use a triangular test. In 'descriptive organoleptic cup analysis', trained assessors sample three types of coffee, whereby two cups belong to the same coffee type and they have to discriminate the unique coffee type using standard descriptors and define a cup profile (Leroy et al., 2006a). The market value of $C$. canephora has persistently remained lower than that of $C$. arabica, because the Arabica coffees are regarded as having more acidity and less caffeine than the neutral Robusta coffees. Neither, the traditional dry and occasional wet processing methods nor the blending with other coffees, has improved the Robusta coffee quality (UCTF, 2008; 2009). It is most likely that the diploidy nature of $C$. canephora $(2 \mathrm{n}=2 \mathrm{x}=22)$ compared to the tetraploidy level of $C$. arabica $(2 n=4 x=44)$ is responsible for the weak, neutral and pronounced bitterness in Robusta coffee (Bertrand et al., 2003). Beverage quality, which is under the influence of the level of biochemical compounds, is the factor that determines the price of coffee in the export markets. Higher levels of chlorogenic acid and caffeine found in Robusta than in the Arabica coffee are reported to impart unfavourable effects on the beverage quality whereas the lower trigonelline and sucrose levels might be responsible for the neutral taste in the Robusta coffee (Clifford, 1985; Ky et al., 2001a, b).

Robusta grows naturally in Uganda and constitutes $80 \%$ of the total area under coffee. Most of the Robusta genotypes are the local landrace cultivars, namely the 'erecta' and 'nganda' with improved varieties being grown on a limited scale. These coffee types offer a wide genetic diversity for an array of many agronomic characteristics, including biochemical 
compounds (Aluka et al., 2006). Nevertheless, cup profiles and organoleptic factors that are critical to market prices have so far not been described (UCTF 2008; 2009).

For many years in Uganda, the organoleptic qualities of Robusta coffee were assessed through the protocols developed for Arabica coffee. These protocols have been misleading since the biochemical levels in the two species are different. The development of an independent sensory assessment for Robusta using a standardized protocol might help to unveil cup traits that are specific to Robusta. A preliminary organoleptic analyses was conducted by Aluka et al,. (2006) on 40 accessions of Robusta coffee. Earlier, Moschetto et al., (1996) had established that there were significant differences in aroma, acidity, body and bitterness, between the Guinean and Congolese Robusta coffee groups. There have been efforts in the past to improve the genetic factors that influence cup quality in Robusta coffee. Needless to say, the genotypic constitution of the biochemical compounds that determine quality are greatly influenced by environmental factors such as altitude, rainfall and temperature (Cannell, 1985; Clifford et al., 1985; Decazy et al., 2003). Montagnon et al., (1998) reported that biochemical compounds and organoleptic cup traits could be improved without affecting yield. Positively significant correlation coefficients between preference and factors such as acidity and aroma have been reported in hybrids and commercial clones (Moschetto et al., 1996). In other efforts, $C$. canephora quality improvement has been attempted through interspecific crosses with $C$. congensis and $C$. liberica, the latter two species having larger bean size and a better organoleptic quality (Moschetto et al., 1996 ; Yapo et al., 2003). In Uganda, introduction of Arabusta hybrids between $C$. arabica and C. canephora with improved cup qualities have not been adopted because of farmers continued preference for $C$. canephora agronomic traits (Ky et al., 1999). Recently, the opportunity to introgress farmer and consumer preferences from Arabica coffees into Robusta, appears greater than before, since genetic maps for coffee quality have been developed and even Quantitative trait loci (QTL) for biochemical compounds such as trigollenine and chlorogenic acid have been mapped (Leroy et al., 2011;Campa et al., 2003).

But because, up to now, there has been no considerable research in Uganda to improve Robusta organoleptic quality, this work set to analyze the structure of organoleptic cup variability and to develop cup profiles that would be useful in demarcating potential market niches.

\section{Materials and Methods}

\subsection{Samples and Green bean preparation prior to cupping}

A representative sample size of 206 Robusta coffee genotypes was selected from an initial germ-plasm based on the Mahalanobis distances of major bean biochemical compounds that influence cup quality derived from Near Infra Red Spectroscopy (NIRS) fingerprint (Aluka et al., 2006). The 206 selected samples comprised of on-farm collections from 21 districts and also from Kawanda and Kituza Robusta germ-plasm collections. Three other samples with proven cup test characteristics from Uganda Coffee Development Authority (UCDA) were included as controls. 300 grams of green beans were measured out and randomly selected for evaluation. 
A trained Robusta roaster from Uganda Coffee Development Authority (UCDA) roasted 10 clean green bean samples a day comprising of 55-120 grams each, at temperatures that exceeded $200^{\circ} \mathrm{C}$. The roasted coffee was left to cool until room temperature and stored overnight in a cool dry place free from other odours and air flow to minimize contamination. The silver skin was removed by rubbing softly and by use of a motorized blowing machine. Three separate measurements of 14.0 grams each from the roasted sample were ground to medium size using a motorized grinding machine and the powder was kept in three different cupping glasses ("rocks" glass with thick walls) and covered with a paper lid. The grinding machine was constantly cleaned with a ground powder of the same sample in advance. The three glasses with coffee powder were arranged in a triangular manner (1, 2 per row) for evaluation. Roasted and green coffee of each sample were placed beside a triangle tip and covered until after the cupping session was over to provide additional comments about the cup based on bean appearance.

\subsection{Data scoring}

Data collection was scored by three professional Robusta coffee organoleptic cup testers from Uganda Coffee Development Authority (UCDA). The cupping exercise used protocols developed by ICO (1991), The Coffee Quality Institute of America (CQIA) and Specialty Coffee Association of America (SCAA). Biochemical flavours that influence Robusta cup quality were subjectively detected quantified and described using the cupping vocabulary.

Ground coffee fragrance was rated on a numeric scale of 1-10 (1=least perceived and $10=$ strongly perceived) based on the cupper's previous experience. To avoid staling and oxidation, a ratio of $8.25 \pm 0.25$ grams of roasted ground powder per $150 \mathrm{ml}$ of boiled water was infused (brewed) within 15 minutes of grinding using boiled mineral water $\left(94^{\circ} \mathrm{C}\right)$ from Rwenzori Beverage Company Limited, Uganda, an International Standards Organization (ISO) 9001-2000 certified Company. The water mineral composition in parts per million (ppm) comprised of Sodium (9.2), Potassium (2.5), Fluoride (0.6), Chloride (4.0), Copper (0.005), Magnesium (3.7), Iron (0.04) and Calcium (10.5) with a $\mathrm{pH}$ of 6.9. Organoleptic cup attributes scored included aroma, flavour, aftertaste, salt/acid, bitter sweet, mouth feel, aftertaste and balance. Defective unpleasant smell sensations were also recorded. Cupping spoons and cuppers mouths were rinsed with boiled water between each coffee taste test.

Coffee aroma that pre-evaluates flavour and coffee brightness was perceived by sniffing brewed coffee volatile compounds nasally, holding brewed coffee in the mouth and then swallowing (http://www.coffeeresearch.org/science/aromamain.htm). After 5 minutes of coffee brewing and sufficient cooling, brew flavour and aftertaste was assessed by passing the liqour in the mouth. Mouth-feel or liquor body determined by fiber and fat content in the brew was assessed by comparing the viscosity (weight) and slipperiness (texture) of coffee liquor with that of pure water. The cup brew was perceived as either sweet or bitter and remarks were scored. A brew with equal intensities of flavour, aftertaste, mouth feel and bitter/sweet was quantified as a balanced cup. Aftertaste was the lingering remnant sensation experienced at the back of the throat after swallowing and often changed over time. All flavour attributes in the coffee brew were put together in a single personal judgment of one score guided by past 
experience. Total scores were obtained by summing scores for the different primary attributes. Each cup attribute was evaluated three times as the liquor brew cooled. The scores ranged from $1-100 \%$. Cupping was kept to a maximum of 10 samples per session to minimize accumulation of caffeine in the mouth that adversely alters cupping ability. After the cupping session, coffee samples were uncovered and additional comments were scored based on appearance.

\subsection{Data analysis}

Analysis of Variance (ANOVA) using XLSTAT version 2011.2.05 statistical program was used to compare to organoleptic cup attributes. Means were separated with Tukey Honestly Significant Difference (HSD) at 95\% confidence. Shapiro-Wilk test in XLSTAT version 2011.2.05 statistical program (Addinsoft, Paris, France) was used to establish the non-parametric distribution of the data. Spearman correlation coefficients were used to quantify the strength between a pair of organoleptic cup attributes relationship measured on ordinal scale at $5 \%$ significance level.

Since the sampled cup bean biochemical compounds were assumed not to be normally distributed, the t-test was used to identify any significant differences between means. Bartlett's specificity test in XLSTAT version 2011.2.05 statistical program established the significant differences among correlated phenotypic parameters before performing Principal Component Analysis (PCA). The PCA aggregated genotypes into high internal homogeneity and high external heterogeneity using genetic distances estimated from the Euclidean straight line method (Mohammadi and Prasanna, 2003). Varimax rotation in XLSTAT version 2011.2.05 statistical program was used to improve the principal component plot reliability (Mohammadi and Prasanna, 2003).

Four organoleptic cup diversity groups derived from the PCA were clustered using the K means analysis that categorized genotypes with related cup biochemical compounds beyond PCA analysis (Mohammadi and Prasanna, 2003). A similarity index calculated the distance of each organoleptic character from the average and determined whether the accessions were from the same or different populations. Group means in K clustering were created based on un-weighted paired group mean algorithms. The factorial step discriminant analysis was used to distribute the $\mathrm{K}$ means spatially. The Mahalanobis and Fisher inter-group distances at $95 \%$ probability were calculated using factorial step discriminant analyses to ascertain how the populations were related. The confusion matrix was used to estimate the efficiency of genotype placement among groups. The percentage similarity variance contributed by each of the organoleptic cup attributes to the $\mathrm{K}$ means analysis group formation and mean group abundance was estimated using Bray-Curtis distance measure.

\section{Results}

There were significant differences among evaluators for all organoleptic cup attributes as shown in Table 1. Evaluators 1 and 2, rated accessions as significantly different for fragrance/aroma $(p<0.05)$ and poor cup balance $(p<0.0001)$. Evaluators 2 and 3 detected significant differences in low salt/acidity $(p<0.0001)$ in bitterness/sweetness $(p<0.0001)$ and gave a total low score. 
Evaluator 1 scored low for flavour, mouth feel, and overall cup taste whereas, Evaluator 2 detected significant differences in aftertaste and cup balance.

Table 1. ANOVA of Robusta coffee organoleptic cup attributes by three evaluators

\begin{tabular}{|l|lllll|l|}
\hline Cup attributes & $\mathrm{R}^{2}$ & v.r & F pv & \multicolumn{2}{l|}{ Evaluators } & \multicolumn{2}{l|}{ Coefficient } & \multicolumn{1}{l|}{ F pv } \\
\hline Fragrance/Aroma & 0.03 & 9.89 & $* * *$ & EV1,EV2 & $0.13,0.4$ & $* *, * *$ \\
Flavour & 0.03 & 10.66 & $* * *$ & EV1 & -0.24 & $* *$ \\
Aftertaste & 0.05 & 15.85 & $* * *$ & EV2 & 0.23 & $* * *$ \\
Salt/acidity & 0.20 & 76.90 & $* * *$ & EV2,EV3 & $-0.55,-0.54$ & $* * *, * * *$ \\
Bitterness/sweetness & 0.19 & 70.39 & $* * *$ & EV2,EV3 & $-0.62,-0.59$ & $* * *, * * *$ \\
Mouth-feel & 0.06 & 18.38 & $* * *$ & EV1 & -0.30 & $* *$ \\
Cup balance & 0.14 & 50.73 & $* * *$ & EV1,EV2 & $-0.26,-0.22$ & $* * *, * * *$ \\
Overall & 0.05 & 14.57 & $* * *$ & EV1 & -0.25 & $* * *$ \\
Total score & 0.09 & 31.73 & $* * *$ & EV2,EV3 & $-0.64,-0.67$ & $* *, * * *$ \\
\hline Fstat;F2, 621 & & & & & & \\
\hline
\end{tabular}

Key to Table $1 ; * *, * * *$ significant at $\mathrm{p}<0.05,0.0001$ levels of probability

$\mathrm{R}^{2}=$ coefficient of multiple determination; v.r = variance ratio; $\mathrm{F}$ pv $=$ Fisher's probability value, p; coefficient=model coefficient; $E V=$ =valuator; EV1=Fidel; EV2=Beatrice; EV3=Rita; F Stat; F2, 621 = Fisher statistics, 2 degrees of freedom for factor (evaluators) =F2 and 621 degrees of freedom for cup attribute variables

The least rated organoleptic cup attribute was salt/acid with a minimum score of 6.0, maximum value of 7.67 and mean rating of 6.80 (Table 2). The highest was for cup balance with a minimum score of 6.5 and mean rating of 7.43 was for cup balance. Aftertaste and fragrance/aroma had the most organoleptic cup variance range of 2.34 and 2.0 respectively while bitter/sweetness and cup balance with variance ranges of 1.50 and 1.67 were the least (Table 2) an indication that these two latter attributes were the most difficult to measure. $61-70 \%$ of all the accessions were of average grade, whereas $71-80 \%$ and $81-90 \%$ were of high quality and fine grades respectively (Table 3 ).

Table 2. Variability of organoleptic cup attributes derived from 206 genotypes

\begin{tabular}{|l|lllllll|l|}
\hline & Faroma & Flavour & Aftaste & Salt/acid & B/sweet & Mth feel & Cup-Bal & Overall \\
\hline Minimum & 6.33 & 6.33 & 6.33 & 6.0 & 6.33 & 6.25 & 6.5 & 6.17 \\
Maximum & 8.33 & 8.33 & 8.67 & 7.67 & 7.83 & 8.17 & 8.17 & 8.33 \\
Mean & 7.32 & 7.42 & 7.36 & 6.80 & 7.09 & 7.30 & 7.43 & 7.41 \\
V range & 2.0 & 2.0 & 2.34 & 1.67 & 1.50 & 1.92 & 1.67 & 2.16 \\
S.E & 0.03 & 0.02 & 0.03 & 0.02 & 0.02 & 0.02 & 0.02 & 0.02 \\
\hline
\end{tabular}

Key to Table 2: S.E-standard error ; Faroma, fragrance-Aroma; Aftaste-aftertaste; 
Salt/acid-saltiness and acidity; B/sweet-bitter sweet ; Mth feel-mouth feel; Bal-cup balance

Only $50 \%$ of the accessions were of fair grade. There were no accessions that could be described as belonging to the fine grade in terms of being high in salt/acidity and bitterness/sweetness (Table 3) and even in the very good category, only 39 accessions could be classified as being high salt/acidity, the majority being in the average category. In terms of bitterness/sweetness, about half of the genotypes could be categorized as average or very good grades (Table 3). Most of the accessions belonged to the very high grade and scored high for fragrance/aroma, flavour, aftertaste, mouth feel, balance and overall cup attributes (Table 3).

Table 3. Organoleptic cup attribute score rating for 206 coffee genotypes

\begin{tabular}{|c|c|c|c|c|}
\hline \multirow[b]{2}{*}{$\begin{array}{l}\text { Organoleptic } \\
\text { attributes }\end{array}$} & \multicolumn{4}{|c|}{ Accession score rating } \\
\hline & $\begin{array}{l}\text { Fair } \\
50-60 \%\end{array}$ & $\begin{array}{l}\text { Average } \\
61-70 \%\end{array}$ & $\begin{array}{l}\text { Very good } \\
71-80 \%\end{array}$ & $\begin{array}{l}\text { Fine } \\
81-90 \%\end{array}$ \\
\hline Fragrance/aroma & 0 & $50(32.7 \%)$ & $153(64.9 \%)$ & $5(2.4 \%)$ \\
\hline Flavour & 0 & $29(14 \%)$ & $173(83.1 \%)$ & $6(2.9 \%)$ \\
\hline Aftertaste & 0 & $43(20.7 \%)$ & $155(74.5 \%)$ & $10(4.8 \%)$ \\
\hline Salt/acid & 0 & $169(81.3 \%)$ & $39(18.7 \%)$ & 0 \\
\hline Bitter /sweet & 0 & $107(51.4 \%)$ & $101(48.6 \%)$ & 0 \\
\hline Mouth feel & 0 & $48(23.1 \%)$ & $156(75 \%)$ & $4(1.9 \%)$ \\
\hline Balance & 0 & $19(9.1 \%)$ & $186(89.5 \%)$ & $3(1.4 \%)$ \\
\hline Overall assessment & 0 & $27(13 \%)$ & $175(84.1 \%)$ & $6(2.9 \%)$ \\
\hline
\end{tabular}

All organoleptic cup attributes had a significant and positive correlation with overall cup assessment as indicated in Table 4. Cup balance ( $\mathrm{r}=0.75)$, mouth feel $(\mathrm{r}=0.73)$, flavour, $(\mathrm{r}=0.72)$ and aftertaste $(\mathrm{r}=0.62)$, were all highly positively significantly correlated with the overall score but aroma $(\mathrm{r}=0.21)$ salt/acid $(\mathrm{r}=0.41)$ and bitterness/sweetness $(\mathrm{r}=0.54)$ though positively correlated with overall score had lower coefficients. Fragrance/aroma was not correlated with salt/acid and with bitterness/sweetness taste.

Table 4. Relationships among coffee organoleptic cup attributes in 206 genotypes

\begin{tabular}{|llllllll|}
\hline & Faroma & Flavour & Aftaste & Salt/acid & Bsweet & Mthfeel & Balance \\
\hline Faroma & - & & & & & & \\
Flavour & $0.32^{* *}$ & - & & & & & \\
Aftaste & $0.27^{* *}$ & $0.75^{* * *}$ & - & & & & \\
Salt/acid & 0.10 & $0.34^{* * *}$ & $0.25^{* *}$ & - & & & \\
B/sweet & 0.13 & $0.43^{* * *}$ & $0.33^{* * *}$ & $0.31^{* * *}$ & - & & \\
Mthfeel & $0.21^{*}$ & $0.60^{* * *}$ & $0.54^{* * *}$ & $0.31^{* * *}$ & $0.37 * * *$ & - & \\
Balance & $0.24^{* *}$ & $0.61^{* * *}$ & $0.57^{* * *}$ & $0.51^{* * *}$ & $0.42^{* * *}$ & $0.64 * * *$ & - \\
Overall & $0.25^{* *}$ & $0.72^{* * *}$ & $0.62^{* * *}$ & $0.41^{* * *}$ & $0.54^{* * *}$ & $0.73^{* * *}$ & $0.75^{* * *}$ \\
\hline
\end{tabular}


Key to Table 4; *, **, *** $=$ p> $0.05,0.003,0.0001$ levels ; Faroma-Fragrance/Aroma ;Aftaste-Aftertaste ; Mthfeel-mouth feel ;salt/acid-salt/acidity ; Bitter/sweet-bitterness/sweetness

As shown in Figure 1, cup balance was regarded the most important organoleptic attribute in overall cup assessment $\left(\mathrm{R}^{2}=0.90\right)$. Flavour and mouth feel were rated second in overall cup assessment. Fragrance/ aroma and salt/acidity were considered the least important attributes in overall cup test respectively $\left(\mathrm{R}^{2}=0.22,0.42\right)$.

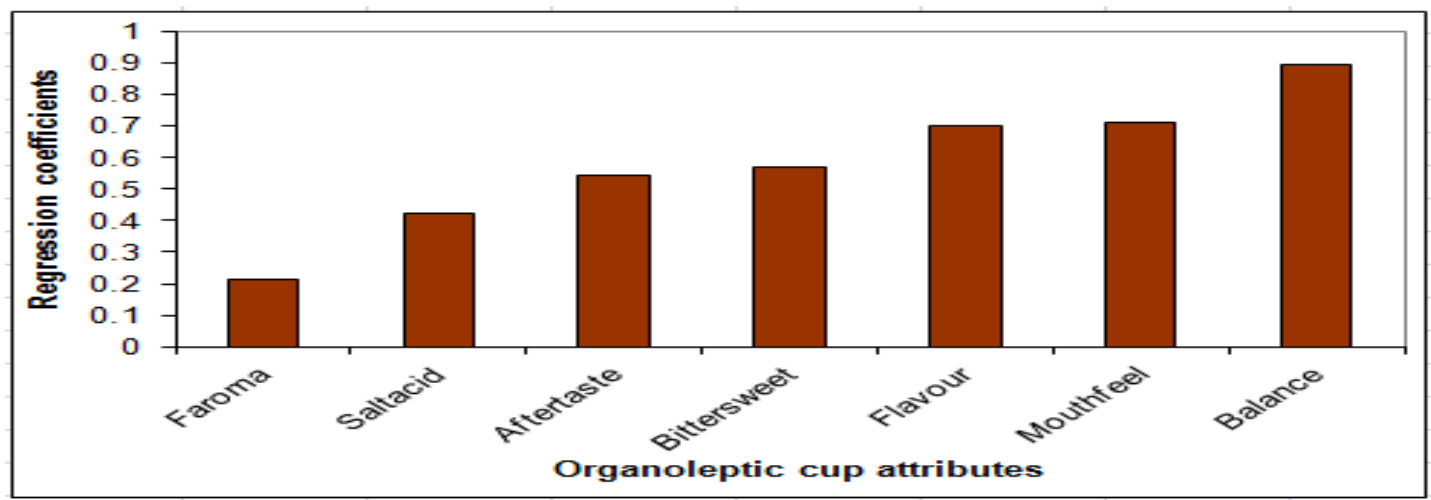

Figure 1. Contribution of organoleptic cup attributes to overall organoleptic cup assessment using regression coefficients

'Erecta' and 'nganda' landraces had a more salty/acidic organoleptic cup as compared to the commercial and hybrid types shown by Figure $2 \mathrm{a}$. The 'erecta' types had a more bitter/sweet cup than hybrids, and it appears that salt/acidity and bitter/sweet were closely associated with each other in all cultivars, a fact also confirmed by the positive significant correlation coefficient in Table $3(\mathrm{r}=0.31)$. Altitude range of 1301-1400 metres above sea level (m a s 1 ) produced better aftertaste while elevation of 1401-1500 metres above sea level had reduced aftertaste (Figure 2 b). Robusta coffee acidity mean values were highest at 1201-1300 metres above sea level and lowest at 1501-1600 metres above sea level. Organoleptic cup balance was highest at elevation 1301-1400 metres above sea level and lowest at 1501-1600 metres above sea level.

(a)

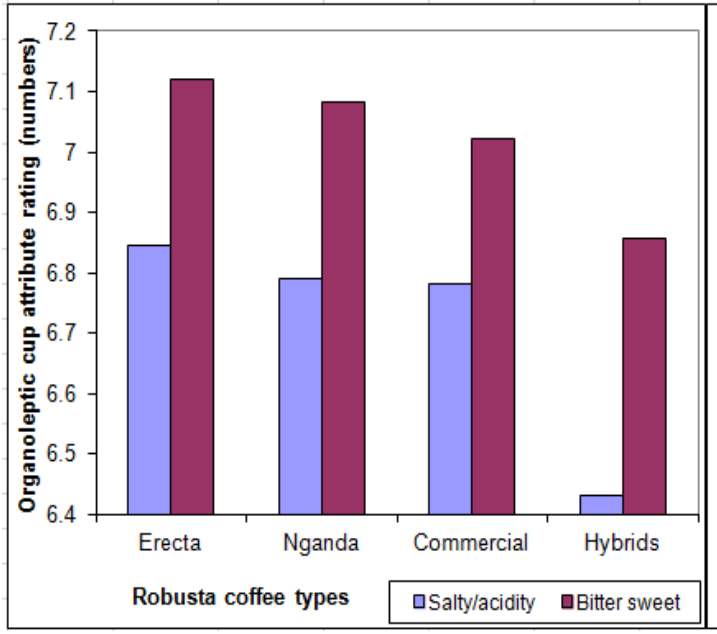

(b)

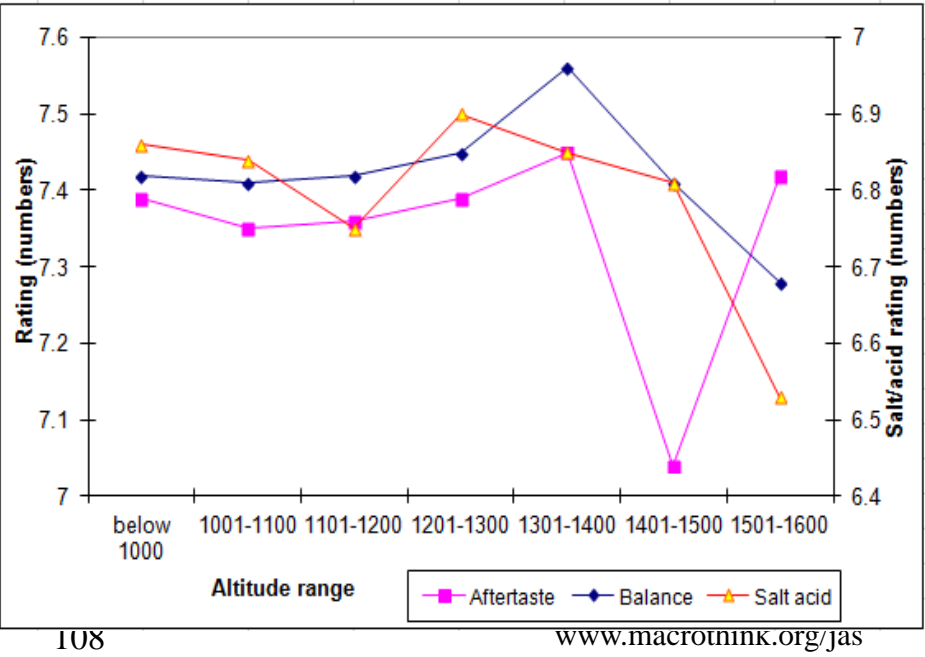




\section{Macrothink}

were devoid of these attributes. A third group comprised of genotypes that had better cup balance, salt/acidity and bitter/sweet attributes while opposite to this group, the genotypes, in group 4 were limited in those attributes.
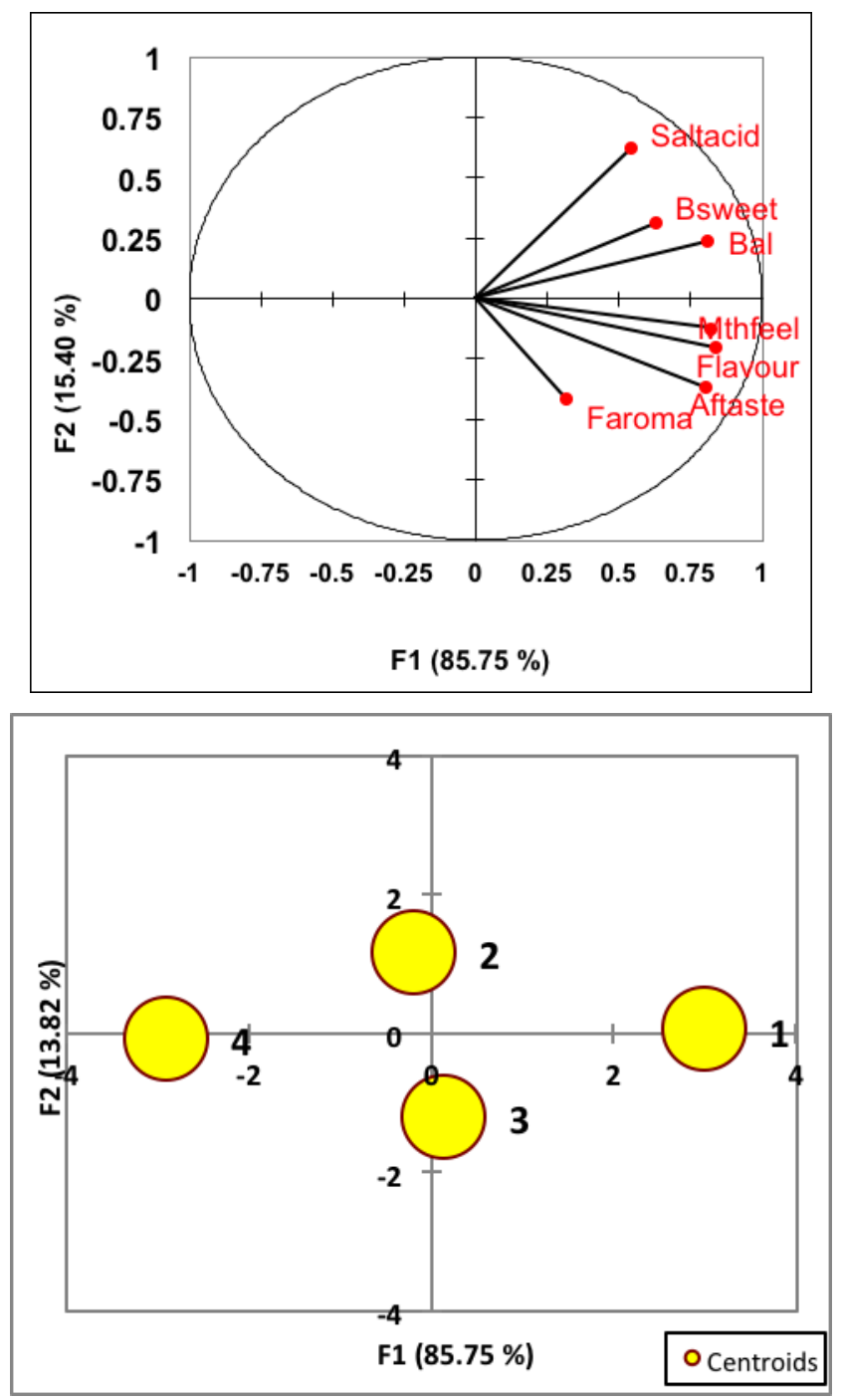

Figure 3(a) Principal Component Analysis of Robusta seven coffee organoleptic cup diversity in 206 accessions (b) Factorial Discriminant Analysis of the groups

Differences in organoleptic cup attribute diversity in the four groups is shown in Figures 4a-g. Except for fragrance/aroma where group 3 had the highest mean, the rest of the attribute means were highest in group 1 and lowest in group 4 (Figure $4 \mathrm{a}-\mathrm{g}$ ). Attribute group means were placed either above or below the median. All attribute sub-groups variance and inter quartile range values varied pointing out the different levels of variation present among in the groups as shown in Figures 4a-g. 
$\mathrm{a}$

$\mathrm{b}$

$\mathrm{c}$

$\mathrm{d}$

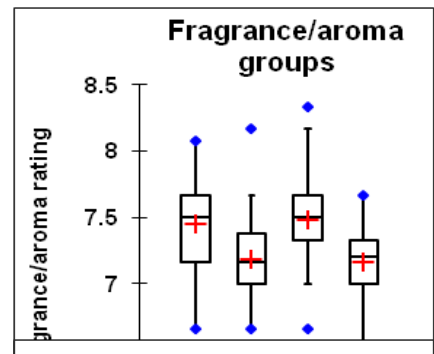

Bitter/sweet groups
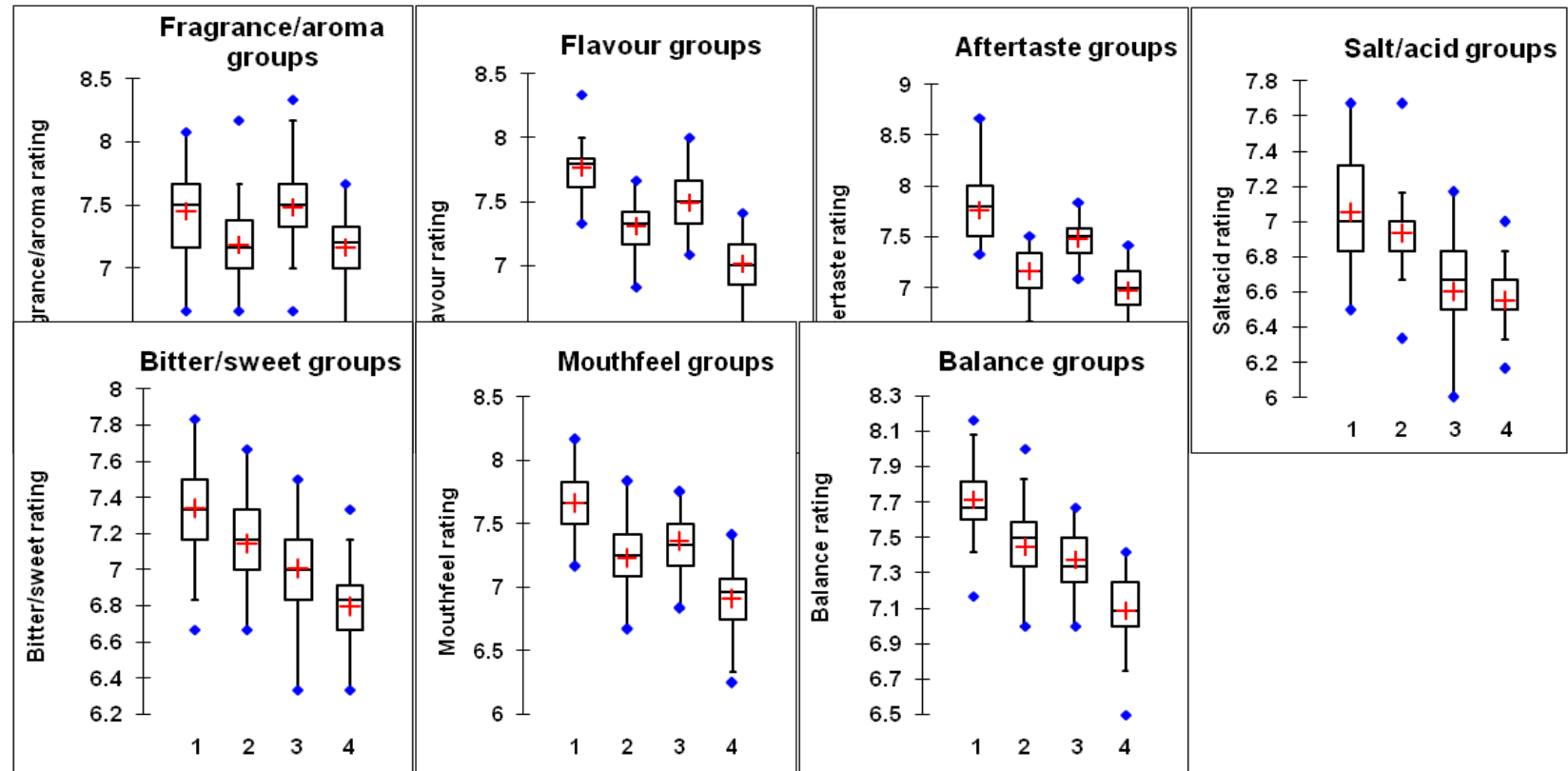

e
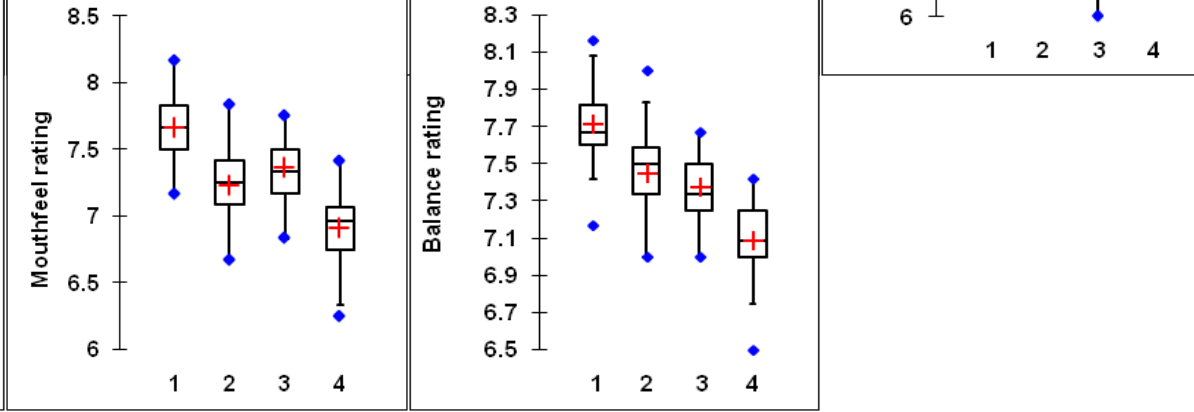

Figures 4 (a-g). Description of organoleptic cup attribute diversity groups

All Mahalanobis distances were greater than 3, implying that the groups were different populations (Table 5). Equally, all the Fisher distances were significantly different, implying that the formed groups had different organoleptic cup attributes. The Fisher as well as the Mahalanobis data indicated that the longest distances was between group 1 and 4 while the shortest was between group 2 and 3. The distance between group 1 and 2 was greater than that between group 1 and 3.

Table 5. Mahalanobis and Fisher distances for organoleptic cup attribute groups

Fisher distances

\begin{tabular}{|l|l|l|l|l|l|l|l|l|}
\hline Gp & 1 & 2 & 3 & 4 & 1 & 2 & 3 & 4 \\
\hline 1 & 0 & & & & 0 & & & \\
\hline 2 & 11.81 & 0 & & & $46.18^{*}$ & 0 & & \\
\hline 3 & 9.80 & 5.76 & 0 & & $36.35^{*}$ & $22.31^{*}$ & 0 & \\
\hline 4 & 35.73 & 9.10 & 11.14 & 0 & $117.04^{*}$ & $30.97^{*}$ & $36.20^{*}$ & 0 \\
\hline
\end{tabular}

Fisher's distances marked * were significant at $\mathrm{p}<(0.0001)$

Figures 5 ( $a$ and $b$ ), further confirms the results of Figures 3 ( $a$ and $b$ ) and those in Table 6. In Table 5 (a), factor 1 of the PCA, contributed $38.85 \%$ variance while factor 2 contributed $12.52 \%$ variance when altitude and age were also considered alongside accessions. Age and altitude accounted for the organoleptic attributes variance in all accessions. Group 1 comprised of genotypes that were superior in all organoleptic cup attributes (Figure $5 \mathrm{a}, \mathrm{b}$ ). Group 2 had more acidity, sweet mouth feel while group 3 genotypes had better fragrance and aroma, aftertaste, balance and flavour (Figure $5 \mathrm{a}, \mathrm{b}$ ). Genotypes that were inferior in all 
organoleptic cup attributes were placed in group 4 (Figure $5 \mathrm{a}, \mathrm{b}$ ). Although fragrance and aroma increased with altitude, very high elevations and old trees had reduced organoleptic quality. The longest distance was between group 1 to 4 while group 2 and 3 distances were in between 1 and 4 groups.
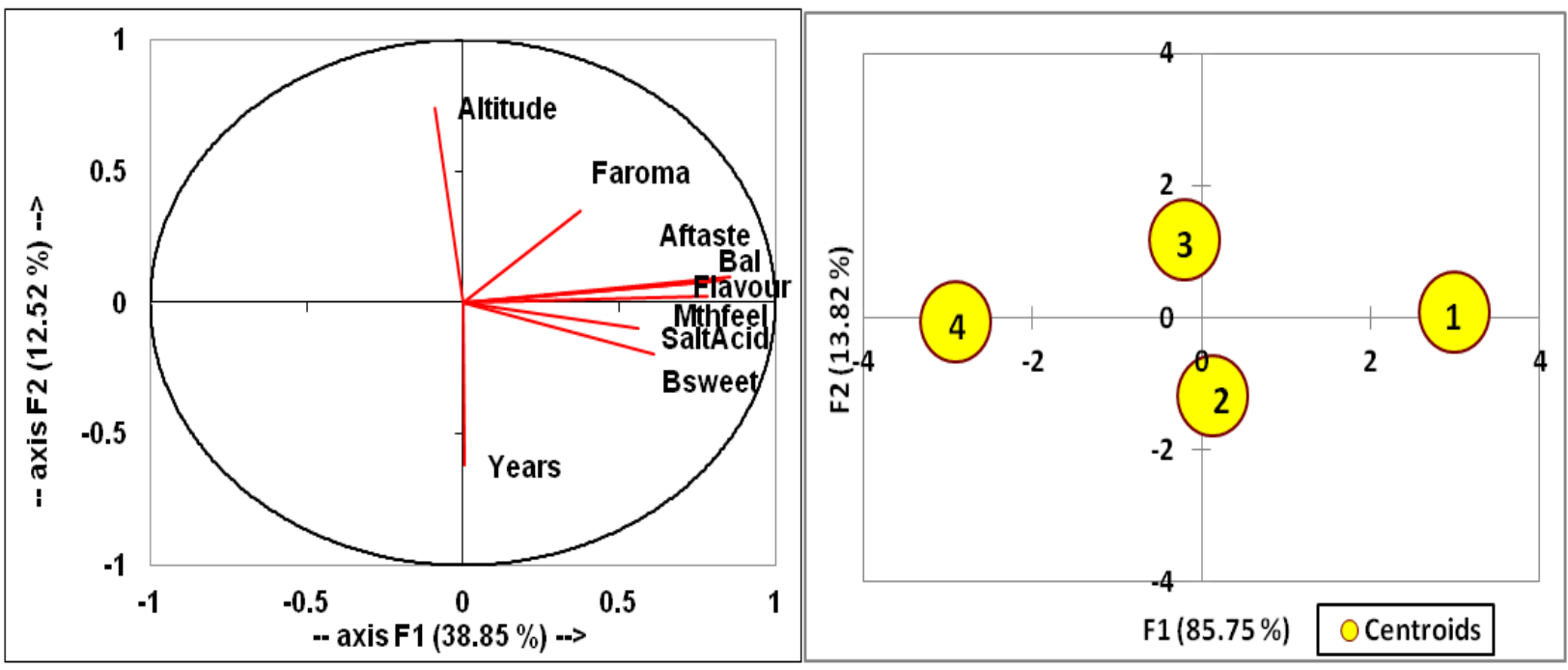

Figure 5 (a). Organoleptic cup attribute relationships with altitude and tree age as shown by the Principal Component Analyses (b) Organoleptic cup attribute groups from a factorial step discriminant analysis

Table 6 also confirms that all the groups were correctly placed. Correct group placement ranged from $88.10-96.23 \%$ with an average of $92.79 \%$ (Table 6).

Table 6. Estimated correct genotype group placement and pair wise distance comparison for farm Robusta organoleptic cup attributes using confusion matrix

\begin{tabular}{|l|lllll|l|}
\hline from $\backslash$ to & 1 & 2 & 3 & 4 & Total & $\%$ correct \\
\hline 1 & 50 & 2 & 2 & 0 & 54 & $92.59 \%$ \\
2 & 1 & 54 & 4 & 0 & 59 & $93.22 \%$ \\
3 & 0 & 1 & 52 & 0 & 53 & $96.23 \%$ \\
4 & 0 & 1 & 0 & 41 & 42 & $88.10 \%$ \\
\hline Total & 51 & 58 & 58 & 41 & 208 & $92.79 \%$ \\
\hline
\end{tabular}

All the organoleptic attributes measured had more or less the same mean abundance that ranged from 0.35 for cup balance to 0.47 for aftertaste (Table 7). But cup balance had a cumulative percentage variance of $100 \%$ meaning that the groups were distinctly different for this trait and less so for aftertaste (Table 7). 
Table 7. Pooled contribution of variance for Robusta coffee organoleptic cup attributes and mean group content derived from Bray-Curtis distance measure

\begin{tabular}{|l|ll|llll|}
\hline \multirow{2}{*}{ Variable } & \multicolumn{4}{|l|}{ Variance } & \multicolumn{5}{|l|}{ Mean group abundance } \\
\hline & Contribution & \multicolumn{1}{l}{ Cumulative \% } & 1 & 2 & 3 & 4 \\
\hline Aftaste & & & & & & \\
Mthfeel & 0.47 & 16.49 & 7.76 & 7.16 & 7.47 & 6.97 \\
Flavour & 0.43 & 31.43 & 7.66 & 7.22 & 7.36 & 6.91 \\
Faroma & 0.43 & 46.33 & 7.77 & 7.31 & 7.49 & 7.02 \\
Salt/acid & 0.42 & 60.95 & 7.45 & 7.18 & 7.48 & 7.16 \\
B/sweet & 0.39 & 74.53 & 7.05 & 6.93 & 6.6 & 6.55 \\
Cup Balance & 0.37 & 87.63 & 7.34 & 7.14 & 7.01 & 6.79 \\
\hline
\end{tabular}

\section{Discussion}

The protocol used to evaluate farm Robusta coffee organoleptic cup attributes was able to detect, differentiate and segment organoleptic cup differences using their technical experience (Tables $1 ; 2 ; 3$ ). The significant differences found in evaluator organoleptic cup scores reflect the subjective individual preference enriched by past experience. Individual cup taste perception and preference for varietal specific characters such as acidity, body, aroma, flavour and taste that constituted the nature and scoring of the brew implied that markets too vary and are specific. Coffee drink preference is personal and consumers have a specific taste according to their nationality, which makes it further difficult to define organoleptic cup quality (Leroy et al., 2006). Furthermore, cup flavour in roasted coffee is reported to constitute of over 800 multiple aromatic compounds and individuals perceive them differently (Wintgens, 2004). Other factors that might also have contributed to liquor differences among the accessions but were not measured in this study include, date of harvesting, processing and storage.

Organoleptic cup characteristics were variable as shown in Table 2. Overall salt/acidity had the least cup grading, an attribute that confers a low grade rating for Robusta coffee (Prakash et al., 2005), despite some cultivars being achieving premium grade in all other attributes. Of the 206 genotypes, $81 \%$ had average liquor salt/acidity of the usual good quality while $19 \%$ were of very good premium grade.

Most of the assessed attributes were positively significantly correlated to each other as shown by Table 4, meaning that there is opportunity to improve most of the desirable organoleptic characters simultaneously. The overall cup quality was highly positively significantly correlated with cup balance $(r=0.75)$, mouth feel $(r=0.73)$ and flavour $(r=0.72)$ than with aroma $(\mathrm{r}=0.32)$ and salt/acidity $(\mathrm{r}=0.41)$. However, cup acidity had positive significant correlation coefficients with a sweet cup $(\mathrm{r}=0.31)$, good mouth feel $(\mathrm{r}=0.31)$, cup balance $(\mathrm{r}=0.51)$ and overall cup ( $\mathrm{r}=0.41$ (Table 4$)$ implying that acidity is an important determining factor by consumers. Cup balance accounted for most to the total variance 
(Figure 1) making it, a major organoleptic trait in Robusta coffee and a priority in quality improvement. This fact is further supported by the results in the PCA analysis (Figures 3a, b; $4 \mathrm{a}-\mathrm{g}$ and $5 \mathrm{a}, \mathrm{b}$ ) and by the non-Euclidean dissimilarity cumulative variance shown in Table 7. Cup balance stands out as one trait that could be targeted for improvement as it consistently demonstrated larger variance and diversity than the rest of the attributes. Aroma/fragrance on the other hand was a lesser variable organoleptic trait. Aroma was not at all correlated with acidity or sweetness in this study, though Moschetto et al., (1996) reported linear correlation coefficients between preference and acidity and aroma in Robusta coffee hybrids and in commercial clones. In this study, cup balance and aftertaste were at the highest levels at altitudes between 1301-1400 metres above sea level whereas acidity was on the decline (Figure 2 b). At higher altitudes of 1500 metres above sea level and above, cup balance and acidity levels decreased but aftertaste levels were on the increase (Figure $2 \mathrm{~b}$ ). Decazy et al., (2003) also supported the idea that high sensory quality is associated with altitude, which can be a criterion for selecting genotypes with high levels of salt/acidity (Leroy et al., 2006b).

The 'nganda' and 'erecta' landraces had significantly more acidity and were more bitter/sweeter than the research elite commercial and hybrid types (Figure 2). As suggested by (Bertrand et al., 2006; Dessalegn et al., 2008), selection for vigour and larger seeds in the hybrids and in the commercial types may have led to reduced variability for acidity and sweetness.

The four categories of organoleptic cup attributes obtained from the PCA in Figures 3 and 5 a, b) reflected the combined effect of genotype and genotype $\mathrm{x}$ environment variances. Group 1 (Figures 5, a, b) regarded as having the best brew had the highest ratings for fragrance/aroma, flavour, aftertaste, acidity, sweetness, mouth-feel and cup balance. Group 1 was also influenced by altitude whereas group 2 which was defined by mouthfeel, acidity and bitter/sweetness was also mostly influenced by age. All the variances in groups 3 and 4 were entirely due to altitude and age. Table 7 confirmed that indeed group 1 was the most superior in organoleptic qualities and group 4, the least. The Malanobis and Fisher distances in Table 5, show that the differences between group 1 and 4 that translate to genetic distances, again implied that these two groups of genotypes are distantly related. The fact that four groups comprised of genotypes from different locations that were far apart with varying ecologies and crop husbandry practices, suggest that organoleptic variability was not restricted to any site or location (Figures, 3,4 5; Table 4) but was influenced by both genotype and the environment (Leroy et al., 2006b). The diverse organoleptic cup characteristics (Table 2) coupled with a wide geographical distribution (Table 4; Figures 2 ; 3) provide immense variability among Ugandan grown landraces of coffee that can not only be traded to diverse markets but that can be selected for quality and other desirable agronomic traits.

\section{Conclusion}

Ugandan Robusta coffee was characterized into four distinct organoleptic cup groups using the principle component and factorial step discriminant analyses. Organoleptic cup differences based on Robusta coffee types were detected. About $84 \%$ of Robusta coffee 
produced in Uganda was of the premium grade while $13 \%$ was of the average grade and $3 \%$ of the fine grade. Genotypes with low caffeine, high acidity and superior organoleptic cup attributes exist among 'nganda' and 'erecta' land races and can be identified. Cup balance was the most important organoleptic trait in determining overall cup.

\section{Acknowledgement}

We thank NARO for granting Pauline Aluka study leave to do this work and also acknowledge financial support from USDA. We thank the Ugandan Robusta coffee farmers for providing the ripe beans used in this study, field extension officers for identifying sample farms and the evaluators, for their expertise on coffee cupping. We also thank Mr. Brian Isabirye for his help in data analysis.

\section{References}

Aluka, P., Musoli, P., Curbry, P., Davrieux, F., Ribeyre, F., Guyot, B., De Bellis, F., Pinard, F., Kyetere, D., Ogwang, J., Dufour, M., \& Leroy, T. (2006). Proceedings of the 21st International Conference on Coffee Science, Montpellier, France, 93.

Bertrand, B., Vaast, P., Alpizar, E., Etienne, H., Davrieux, F., \& Charmetant, P. (2006). Comparison of bean biochemical composition and beverage quality of Arabica hybrids involving Sudanese-Ethiopian origins with traditional varieties at various elevations in Central America. Tree Physiology, 26(9), 1239-48.

Campa, C., Noirot, M., Bourgeois, M., Pervent, M., Ky, C. L., Chrestin, H., Hamon S., \& De Kochko, A. (2003). Genetic mapping of a caffeoyl-coenzyme A 3-0-methltransferase gene in coffee trees: Impact on chlorogenic acid content. Theor. Appl. Genet., 107, 751-756.

Cannell, M. G. R. (1985). Physiology of the coffee crop. In N.M. Clifford \& K.C. Willson (Eds), Coffee: Botany, Biochemistry and Production of Beans and Beverage (pp 108-134). London, Croom Helm.

Clifford, M. N. (1985). Chemical and physical aspects of green coffee and coffee products, In M.N. Clifford, \& K. C. Willson (Eds), Coffee: botany, biochemistry and production of beans and beverage, ( pp 305-374), Connecticut, USA, Westport, Avi Publishing Company.

Decazy, F., Avelino, J., Guyot, B., Perriot, J. J., Pineda, C., \& Cilas, C. (2003). Quality of different Honduran coffees in relation to several environments. Journal of Food Science, 68, 2356-2361.

Dessalegn, Y., Labuschagne, M. T., Osthoff, G., \& Herselman, L. (2008). Genetic diversity and correlation of bean caffeine content with cup quality and green bean physical characteristics in coffee (Coffea Arabica L.). Journal of the Science of Food and Agriculture, 88(10), 1726-1730.

International Coffee Organization, (ICO). (1991). Sensory evaluation of coffee: Technical Unit Quality Series, ICO No 9, 209-243. 
Ky, C. L., Louarn, J., Dussert, S., Guyot, B., Hamon, S., \& Noirot, M. (2001a). Caffeine, trigonelline, chlorogenic acids and sucrose diversity in wild Coffea arabica $L$. and $C$. canephora P. accessions. Food Chemistry, 75, 223-230.

Ky, C. L., Guyot, B., Louarn, J., Hamon, S., \& Noirot, M. (2001b). Trigonelline inheritance in the interspecific Coffea pseudozanguebariae x C. liberica var. dewevrei cross. Theor Appl. Genet., 102, 630-634.

Ky, C. L., Louarn, J., Guyot, B., Charrier, A., Hamon, S., \& Noirot, M. (1999). Relations between and inheritance of chlorogenic acid contents in an interspecific cross between Coffea pseudozanguebariae and Coffea liberica var 'dewevrei'. Theor. Appl. Genet., 98, 628-637.

Leroy, T., Ribeyre, F., Bertrand, B., Charmetant, P., Dufour, M., Montagnon, C., Marraccini, P., \& Pot, D. (2006a). Genetics of coffee quality. Braz. J. Plant Physiol., 18(1), 229-242.

Leroy, T., Curbry, P., Durand, N., Dufour, M., De Bellis, F., Jourdan, I., Vieira, L.G., Musoli, P., Aluka, P., Marraccini, P., \& Pot, D. (2006b). Coffea spp. and Coffea canephora diversity evaluated with microsatellites and Single Nucleotide Polymorphism, Lessons from comparative analysis, 21st International Conference on Coffee Science, Montpellier, France, 53.

Leroy, T., De Bellis, F., Legnate, H., Kananura, E., Gonzales, G., Luiz Felipe Pereira, L.F., Andrade, A. C., Charmetant, P., Montagnon, C., Cubry, P., Marraccini, P., Pot, D., \& De Kochko, A. (2011). Improving the quality of African robustas: QTLs for yield and quality related traits in Coffea canephora. Tree Genetics \& Genomes, 1-18. http://dx.doi.org/10.1007/s11295-011-0374-6.

Mohammadi, S. A., \& Prasanna, B. M. (2003). Analysis of Genetic Diversity in Crop Plants; Salient Statistical Tools and Considerations. Crop Science, 43, 1235-1248.

Montagnon, C., Guyot, B., Cilas, C., \& Leroy, T. (1998). Genetic parameters of several biochemical compounds from green coffee. Coffea canephora, Plant Breed., 117, 576-578.

Moschetto, D., Montagnon, C., Guyot, B., Perriot, J. J, Leroy, T., \& Eskes, A. B. (1996). Studies on the effect of genotype on cup quality of Coffea canephora, Tropical Science, 36, 18-31.

Prakash, N.S., Combes, M.C., Dussert, S., Naveen, S., \& Lashermes, P. (2005). Analysis of genetic diversity in Indian Robusta coffee genepool (Coffea canephora) in comparison with a representative core collection using SSRs and AFLPs. Genetic Resources and Crop Evolution, 52, 333-342.

Uganda Coffee Trade Federation, Coffee Year Book. (2009).

Wintgens, J. N. (Ed) (2004). Coffee: growing, processing, sustainable production. A guidebook for growers, processors, traders, and researchers, Weinheim, WILEY-VCH Veriag GmbH \& Co. KGaA, ISBN: 3-527-30731-1.

Yapo, A., Louarn, J., \& Montagnon, C. (2003). Genetic gains for yield after two back crosses of the interspecific hybrid Libusta (Coffea canephora $\mathrm{P}$ x $C$. liberica Bull. Ex Hiern) to $C$. canephora. P. Plant Breed., 122, 288-290. 


\section{Copyright Disclaimer}

Copyright for this article is retained by the author(s), with first publication rights granted to the journal.

This is an open-access article distributed under the terms and conditions of the Creative Commons Attribution license (http://creativecommons.org/licenses/by/3.0/). 